\title{
Nano-biotehnologies and nano-products: achievements and perspectives of researches in animal husbandry and veterinary medicine
}

\author{
V. Vlizlo, \\ academician of NAAS
}

A report by the academician of NAAS V. V. Vlizlo at the general meeting of the National Academy of Agrarian Sciences of Ukraine, March 23th 2017

Results and perspectives of development of nano-biological researches in animal husbandry and veterinary medicine are illuminated. The role of National academy of agrarian sciences of Ukraine and its leading centers of science, in particular the Institute of biology of animals, in formation and execution of the priority directions of nano-biological researches is shown. The modern state and productivity of researches in nano-biotechnology, as well as application of innovative developments and nano-products in animal husbandry and veterinary medicine are analyzed. The necessity of expanding researches in fundamental directions, forming on their basis of the priority state programs is substantiated.

Key words: nano-biotechnology, nano-products, nano-particles, adjuvant, specimens, animal husbandry, veterinary medicine.

The development of nanotechnology was initiated by American physicist the Nobel Prize winner R. Feinman in 1959, suggesting that in the future many materials and devices will be manufactured at atomic or molecular levels and will have unprecedented properties (R. P. Feynman, 1960). However, the term of "nanotechnology" was first introduced by Tokyo University professor N. Tamiguchi in 1974 in his report "On the basic concepts of nanotechnology" (N. Tanigushi, 1974).

Consolidated nanomaterials, nano-polymers, nanobio materials, fullerenes, nanotubes, nanopowders, nanoporous materials, nanostructured liquids (colloids, micelles, gels), pharmacological nanoparticles are being developed today on the basis of nanotechnologies. The development of nanotechnology gave rise to new branches of science, one of which is nanobiotechnology, which is devoted to the study of structural changes, biological and biophysical processes in natural biological objects or their nanobiological analogues.

The United States is a recognized leader in research and development in the field of nanotechnology. Back in 2000, the scientific program "National Nanotechnology Initiative" was developed, which coordinates basic research on this scientific direction. The federal government's annual allocations to nanotechnology amount to approximately $\$ 1$ billion, representing quarter of the corresponding costs of all countries of the world. In addition, the United States is leading the number of research start-up companies, as well as the number of patents and publications in the field of nanotechnology (M. $O$. Kyzym, 2011). The world leader in the total volume of investments, except for the USA, in this area is Japan. EU countries occupy the third place in terms of investments in nanotechnology development. In the EU, the European Commission is the main institution that regulates the nanoindustry and manages various funds and programs at the supranational level. Among these programs there is a program for the development of nanomedicine and ecological chemistry. Annually, the number of scientific publications on nanotechnology in the EU is not less than 12,000 (in USA are about 15,000). And the most cited publications publishing scientific articles in the field of nanotechnology are multidisciplinary - Science in the USA and Nature in Europe. 
More than in 50 countries of the world investments in special nanotechnology development programs are constantly increasing, which has become an important factor in scientific and technological progress and an innovative component of their economy. It is anticipated that the world market for nanomaterials in 2021 should reach $\$ 5.3$ billion, compared with 2016 , when it was $\$ 1.6$ billion.

In the field of fundamental nanotechnology research, Ukrainian scientists take important positions, while in the sphere of their practical implementation and development of nanoindustrial production, our country lags behind the leaders for decades.

In order to accelerate the development of nanobiotechnological research at the National Academy of Agrarian Sciences of Ukraine (NAAS), its Presidium had developed a program of scientific research "Creation and use of nano and biotechnological materials and means in animal husbandry" for 20162020, the main institution of which is the Institute of Animal Biology (IAB) of NAAS. In addition in IAB of NAAS this study is carried out by the Institute of Animals Breeding and Genetics of named after M.V. Zubets, NSC "Institute of beekeepers named after P.I Prokopovich", Bukovyna SAES of NAAS and other institutions. The Department of Veterinary Medicine (NSC "Institute of Experimental and Clinical Veterinary Medicine" of NAAS (IECVM) and the Institute of Veterinary Medicine of NAAS investigate the possibilities of using nano compounds in the manufacture of immunobiological drugs, the influence of nanoparticles on pathogens of diseases, and the toxicological effects of nanomaterials on the body. The tasks of NAAS programs implementation will ensure the development of nanobiotechnological materials and tools, studying their biological effects and efficient use in animal husbandry and veterinary medicine. Today research is conducted to determine the mechanisms of action of nanoparticles and nanocompounds on biological effects in these research institutions.

Nanoparticles and compounds based on them begin to be used in the field of biophysics, molecular biology, proteomics, genetics, in particular for the creation of biomarkers. Magnetic nanoparticles bearing antibodies and DNA fragments have the ability to amplify the signal from numerous biomolecules. This will diagnose the disease in the early stages and more effectively treat various diseases. At the same time, the synthesis of nanoparticles that have the properties of adjuvants and their interaction with antigen (a virus, a bacterium or their fragments) makes it possible to create new generation vaccines. In the field of veterinary medicine, nanoparticles are used in diagnostics to detect viral, parasitic and bacterial pathogens.

Polymeric carriers of pseudopolyaminic acids for the delivery of biologically active substances were created in the IAB of NAAS in cooperation with the National University "Lviv Polytechnic" and are using in the research. Nano-polymer GluLa-DPG-PEG600, which contains glutamic and lauric acids, dipropylene glycol, polyethylene glycol, is created. By electrophoresis in $5 \%$ polyacrylamide gel, the ability of GluLaDPG-PEG600 to bind serum albumin to blood is established. This is a positive characteristic of this polymer as a potential transporter for proteins and drugs, in particular for the production of vaccines, since proteins are the main antigenic components. It is established that said nanopolimer is capable of penetrating through the membrane and entering into sex cells - sperm. Investigations on the depositing of this polymer in the organs of rats revealed its localization in the muscle tissue and its subsequent cleavage with myocytes. Based on the histostructure analysis of animal tissue and organs using luminescent microscopy, it was found that after the intramuscular administration, the GluLa-DPGPEG600-F + BSA Alexa Fluor 555 complex was detected in the liver, brain, kidneys and spleen of animals.

Today there is an active work on the use of pseudopolyaminic acids as carriers of minerals to create premixes and feed additives for animal husbandry.

Nanoparticles are capable of acting as carriers of physiologically active substances, xenobiotics and drugs into the target cells as the basis for the development of the pathological process. Nanocipes, such as phospholipid particles, liposomes, fullerenes, dendrimers, chitosans, nanotubes and others can be used as nanoparticles, which become the "couriers" or "containers". Nanostructurization leads to a reduction in the size of the dosage form and an increase in the level of active substance in the blood. Ukrainian scientists have proved the possibility of transmembrane transport of nanosized complexes and 
particles into cells of bacteria capable of selective accumulation of colloidal particles of $\mathrm{Au}$, as well as the molecular structures and mechanisms responsible for this process.

A new method for detecting cationic oligoelectrolytes conjugates with oligodeoxynucleotides has been developed. The proposed method makes it possible to simplify and cheapen the choice of the best carrier, to visually identify the fact of complexation between the substances that interact. The versatility of this methodological approach is confirmed by interaction with oligodeoxynucleotides of another cationic polymer of natural origin — chitosan (V. V. Vlizlo et al., 2015).

The research on addressing drug delivery conducted at the IAB of NAAS had shown the effectiveness of new polymeric carriers use based on dimethylaminoethyl methacrylate for the transport of antisensoligodeoxinucleotides to mammalian cells (DNA nanotechnology). The results of these studies indicate a decrease in the expression of the physiological prion in the cells of L1210 line by $70-90 \%$. The possibility of successful application of these conjugates for complete suppression of the physiological prion expression in the spleen, small intestine and, most importantly, in the brain of animals is proven. Thus, it has been proved that nano compounds can overcome the blood-brain barrier and affect on to the pathological process in the brain.

The introduction of nanobiotechnological approaches into practice allows for the early diagnosis of diseases. In vitro nanodiagnosis develops in two directions: the use of nanoparticles as markers of biological molecules and the application of innovative nanotechnological methods of measurement. In particular, sensory systems, which are already used in biology, significantly simplify the diagnosis of diseases and allow tracing the interaction between proteins and DNA in real time. DNA chips will be created and improved to allow for the analysis of human or animal genetic information that will help in the development of individual medicines (K. An, 2009).

In the characterization of nanomaterials, solubility, particle size, and permeability through biological membranes are particularly important. The size of the particles should also be optimal, since reducing the size of the particles has its limits not only in terms of technology, but also bioavailability and safety. The desire to get as little particle size as possible cannot be considered justified, as reducing the size can cause their inactivation, rapid withdrawal from the body or manifestation of pathological effects on the body. It is established that the size of nanoparticles at least $5-7$ nanometers in size is physiologically grounded. They are assembled into clusters (associatives), in which the particles do not touch to each other, but are at a distance of $2-3$ nanometers. If the particle sizes are less than $2 \mathrm{~nm}$ their behavior becomes unpredictable. Determination of the genotoxic properties of nanoparticles of metals in vitro and in vivo by the DNA comet method revealed that the Aurum nanoparticles of 20 and $45 \mathrm{~nm}$ in the concentration range of $4-14 \mathrm{~V} \times 10^{5} \mu \mathrm{g} / \mathrm{ml}$ and Argentum of $30 \mathrm{~nm}$ at a concentration of $10^{5} \mu \mathrm{g} / \mathrm{ml}$ did not inhibit physiological processes, but on the contrary, they were activated in probionte bacterial cells. The genotoxic effect on eukaryotic test cells show the 10 and $20 \mathrm{~nm}$ of Aurum nanoparticles of, $20 \mathrm{~nm}$ of Zinc and Kuprum, and 14, 18 and $23 \mathrm{~nm}$ of Ferum in the entire study concentration range.

Argentum nanoparticles ( $\mathrm{Ag}$ ) exhibit antiviral, antibacterial and wound healing effects. Nanodisinfectants based on $\mathrm{Ag}$ nanoparticles have a wide range of biocidal and antiviral activity and a significantly higher toxicity to microbes, viruses and fungi. Thanks to the latest technology for obtaining and depositing nanoparticles, evenly distributed them across the surface of the paper and avoid the formation of agglomerates. The application of nanoparticles of this element to sulfanilamide (in particular, streptocide) modifies the existing drug and results in such positive effects as prolongation and localization of action.

Ag nanoparticles for the destruction of pathogenic microorganisms ere used at the Institute of Veterinary Medicine of NAAS. The research of antifungal and antibacterial properties of the two drugs Sumer silver ( $\mathrm{Ag} \mathrm{i} \mathrm{Cu}$ ) and Yodoselen ( $\mathrm{i} \mathrm{Se}$ ) are conducting at the Institute of Beekeeping of NAAS.

The scientists of the NSC IECVM of NAAS have developed manufacturing technologies and regulations for the use of vaccine inactivated against infectious rhinotracheitis, viral diarrhea and parainfluenza-3 of cattle, using nano-diamonds as adjuvants. The indexes of mitotic activity of cells cultures of FLK-71 and FLK-SBBL under the influence of Fe and Ag nanoparticles were established. The 
cultivation of these sublines with $\mathrm{Ag}$ nanoparticles increases their production of the leukemia virus antigens in 1.6 and 2.0 times respectively. It can be used to improve the technology of manufacturing the domestic import substitute test set for serological diagnosis of cattle leukemia (O. T. Kutsan et al., 2016).

Integrated studies of the influence of $\mathrm{Ag}$ nanoparticles on oocytes maturation, semen storage, fertilization and early embryonic development of rabbits have been carried out at the IAB of NAAS. The conducted studies made it possible to create a liposomal preparation of "Argoton". It is highly effective for the treatment and prevention of endometritis without the use of antibiotics.

Perspective is the use of nanomaterials in analytical biochemistry and diagnostics. It has been established that Ag-hyaluron nanoparticles obtained in the IAB of NAAS have good optical properties (Fig. A). On their basis, a method for determining the content of proteins in tissues, which has a number of advantages compared with the classical methods of analytical biochemistry (Fig. B) has been developed.

The future of nanobiotechnologies is not for free nanoparticles, but for functional nanobio materials, in which the presence of free nanoparticles is minimized, and best of all to zero. Perspective are functional nanobio materials in the form of nano aquachelates of various metals, which exhibit high biological activity and are not toxic, in particular colloidal solutions, aquachelates and hydrated nanoparticles of macro and microelements. In Ukraine, for the first time, nanotechnology for obtaining aqueous solutions of most biotic elements that were not synthesized by chemical methods was developed (V. B. Borysevytch et al., 2009). Nanoaquachelates can be used in agriculture, food industry, medicine, gerontology, for the enrichment of fodder, food and biologically active additives.

At the NAAS institutions researches on the use of nanoaquacitrate macro- and microelements are conducting. The biological activity of $\mathrm{Fe}, \mathrm{Cu}, \mathrm{Zn}, \mathrm{Mg}$ citrates, obtained by the nanotechnology method was evaluated in vitro. Their influence on the culture of human cells and proteins (albumins, immunoglobulins) has been investigated. It was found that $\mathrm{Cu}$ and $\mathrm{Zn}$ nanoparticles exhibited the highest cytotoxic activity in cell culture, and Mg nanoparticles the smallest activity. The largest denaturing activity for human plasma proteins is for Fe nanoparticles, and the smallest for Mg (T. Korolenko et al., 2010).

The important direction of complex nanotechnological and physiological research carried out in the IAB of NAAS is the elucidation of the physiological and biochemical mechanisms of the nanoacquacitrate minerals action in the organism of animals in different periods of ontogenetic development and productive use (R. Ya. Iskra et al., 2014; V. V. Vlizlo et al, 2015). In this direction, feed additives based on citrates of trace elements with high biological activity are developed (V. B. Borysevytch et al., 2009).

Using the introduction of different amounts of nanoacquacitrate minerals into animal diets has been determined the minimum physiologically active and optimal doses for cattle, pigs, rabbits, bees. The influence of these compounds on the content of macro and microelements in tissues and liquids, the formation of immunobiological reactivity in an organism, the state of antioxidant, detoxification, reproductive and immune systems, the growth and development of calves, pigs and rabbits, as well as their role in the treatment and prevention of microelementosis in animals has been investigated. The influence of the nanoacquacitrates of individual elements on the biological value of animal production using the parameters of the chemical composition of milk, meat, the level of fatty acids, microelements and proteins was studied. The results of researches that provided the development of a methodology for studying the biological effects of nanoacquahelates in the organism of animals, as well as their impact on the biological value and quality of animal products were obtained. Certain differences in the action of nanoacquacitrates of $\mathrm{Se}, \mathrm{Cr}, \mathrm{Fe}$ in the organism of animals are established, in comparison with other compounds of these trace elements, due to their increased physiological activity and intensity of absorption in the digestive tract. The toxic doses of 18 elements $(\mathrm{Cr}, \mathrm{Cu}, \mathrm{Fe}, \mathrm{Zn}, \mathrm{Se}, \mathrm{Mn}, \mathrm{Mo}, \mathrm{I}, \mathrm{V}, \mathrm{Bi}, \mathrm{Ni}$, $\mathrm{Mg}, \mathrm{S}, \mathrm{Ge}, \mathrm{Al}, \mathrm{Ag}, \mathrm{Ce}, \mathrm{Si}$ ) and their combinations were determined in the IAB of NAAS, which turned out to be in 6-8 times lower than their mineral salts.

The introduction of $\mathrm{Cr}$, Se, Co and $\mathrm{Zn}$ citrates into the cow diet improved liver function, the exchange of $\mathrm{Ca}, \mathrm{P}$ and vitamin $\mathrm{E}$. Mineral supplement stimulated secretory activity of the mammary gland, increased the average daily milk yield in cows by $3,3-7,8 \%$ (R. S. Fedoruk et al., 2014). 
The complex effect of micronutrient citrates on metabolic processes in the piglet organism during the period of weaning from sows was investigated. The expressed influence of citrates of trace elements (Fe, $\mathrm{Zn}, \mathrm{Mn}, \mathrm{Cu}, \mathrm{Co}$ ) in the lower concentration, in comparison with their inorganic salts, on the parameters of metabolism in the blood of piglets, in particular the growth of antioxidant activity of erythrocytes, their amount, hemoglobin content and total protein has been studied. It is proved that in the conditions of the complex application of these nanocitrates in pigs feeding the adaptive ability and resistance of their organism during the period of weaning from sows increases (R. Ya. Iskra et al., 2014).

The complex study of the influence of citrate ferrum in the composition of the drug of "Nanoferocyte" (TU U 21.2-30995014-009: 2014), created in the Institute of IAB of NAAS, on the fermental and oxygentransport function of the blood and metabolic processes in the piglet organism has been carried out. The effectiveness of using this drug for prevention of alimentary ferrum deficiency anemia has been established. It was found that the introduction of ferrum citrate contributes to the increase of the erythrocytes number and the hemoglobin concentration in the blood, positively affects the ferment binding function of transferrin, stabilizes blood proteins, levels of $\mathrm{Fe}, \mathrm{Cu}, \mathrm{Co}, \mathrm{Mn}$, vitamins $\mathrm{A}$ and $\mathrm{E}, \mathrm{LP}$ products and antioxidant systems indicators (V. V. Vlizlo et al., 2014).

The high efficiency of chromium citrate's ( $\mathrm{Cr}$ (III)) activity was experimentally proved in animal organisms on carbohydrate, protein and lipid metabolism indices, activation of antioxidant, NO-synthase, endocrine and immune systems. It was established that the introduction of citrate chromium into the diet of animals is accompanied by an adjustment effect on the function of the adrenal, thyroid and pancreas.

In pregnant rabbit uterus and sows for the influence of chrome citrate correction of different parts of the metabolism is carried out, in particular stabilizing carbohydrate, lipid and protein metabolism, normalizing antioxidant and immune systems of the organism. Metabolic effective amounts of chrome citrate, which are additionally introducted to animal rations, can be used as recommended doses for the regulation of metabolic processes and the prevention of $\mathrm{Cr}$ (III) deficiency in the body (R. Ya. Iskra, 2013, 2014).

It has been established that nanoaquacitrates of minerals are not only biologically active but also safe for health and are allowed for the enrichment of feed, raw materials and food products. Thus, for the use of $\mathrm{Cr}$, Se and $\mathrm{Ge}$ citrates for bees feeding, a decrease of heavy metals $(\mathrm{Cd}, \mathrm{Pb}$ ) levels was found in both tissues of the whole organism and individual anatomical parts of bees (R. S. Fedoruk, 2014). The changes in the dynamics of lipid level were shown. It contributes to the metabolic accumulation of energy and plastic components and confirms the expediency of using the elements citrates in bees feeding. This ensures their vitality, increases the level of essential microelements, lipid and carbohydrate components in the bees' organism and beekeeping products.

The dynamics and degree of influence of nanoaquacitrates of $\mathrm{Ge}, \mathrm{Se}, \mathrm{Mg}, \mathrm{Cu}$ and $\mathrm{Ag}$ in different doses on the factors of non-specific resistance of the bird organism was established in the NSC IECVM of NAAS (O. T. Kutsan et al., 2016). Due to the addition of nanoferrum citrate to methalloglobulin, improved nanomethalloglobulin had been obtained, which allows the use of a drug for the anemia treatment in newborn piglets and chickens. The positive effect of the complex probiotic-metal-protein drug with the addition of nanoferrum citrate on body weight gain, indices of innate immunity and expression of genes encoding IL-17 $\alpha$, IL-2, IFN- $\gamma$ in the chickens [6] had been proved.

At the IABG named after M.V. Zubets of NAAS for the first time in Ukraine a technology for the conservation and rational use of genetic resources of domestic breeds of pigs using nanomaterials based on highly dispersed silica and sucrose has been developed. The research is carried out within the framework of the PSI 28 in cooperation with the Institute of Surface Chemistry named after O.O. Chuiko of NASU. A cryocollection of 750 doses of sperm and 44 eggs of the Mirgorod pig breed was created. The use of cryocollections will ensure the implementation of measures for the functioning of "virtual gene pool cryostats", which is more profitable, compared to the actual maintenance of animals' herds. In cooperation with breeding and experimental farms of NAAS of pigs domestic breeds breeding, the methodology of cryopreservation of genetic resources of farm animals will be implemented, including a "virtual gene pool cryostat". The developed technology ensured the participation of the IABG named after 
M.V. Zubets in the international FAO project on the preservation of the gene pool of cattle brown Carpathian breed in 2017.

According to the results of nanobiotechnological research and application of nanoproducts, 3 doctoral ( 1 is being prepared) and 6 candidate dissertations ( 11 are being prepared) have been defended and more than 300 scientific papers and 4 methodical recommendations have been published.

At the same time, understanding of the laws of the nanoproducts action requires further study of their properties with the conduct of deep fundamental nanobiotechnological research, which can be successfully implemented in NAAS together with the institutes of the National Academy of Sciences of Ukraine and organizations of other departments.

Therefore, taking into account the results obtained by the institutes of the Zootechnical and Veterinary Medicine Departments of NAAS, scientific research in the field of nanobiotechnology in the future should focus on the following areas: the studying the biological effects of new nano-products effective in animal husbandry and veterinary medicine; the use of nanomaterials for the enrichment and storage of feed; the use of nanoparticles as dietary supplements and regulators of physiological and biochemical functions of the organism; the solving of problems of animals genetics and reproduction on the basis of nanobiotechnologies; the use of nanobiotechnologies and the introduction of nanobiosensors and nanomaterials for laboratory research; the neutralization of xenobiotics, allergens and toxins with the use of nanoparticles; the creation of new nanomaterials and nanoparticles for the diagnosis, prevention and treatment of various diseases.

\section{Conclusions}

Nanobiotechnology is one of the most important areas in biological science, animal husbandry and veterinary medicine, modern agricultural production, as well as food industry, which can become an important basis for further scientific and technological, economic and social development of Ukraine in the near future. An important condition for further advancement in the development of nanobiotechnology in Ukraine can be recognition of this trend as a strategic innovation priority for NAAS scientists with state support at all levels, the complexity of such research, effective protection and preservation of rights to intellectual property objects, their commercialization, timely approbation and introduction of the received developments. 